\title{
$\mathrm{PH} 102$ actuaidad
}

\section{Nace una plataforma para acercar la ciencia ciudadana que se hace en Europa}

Su nombre es EU-Citizen.Science y está disponible desde otoño de 2020. La Fundación Española para la Ciencia y la Tecnología (FECYT) junto al Ministerio de Ciencia e Innovación y otras entidades de hasta trece países europeos tienen la misión de convertirla en un espacio de referencia para el personal investigador, responsables políticos y sociedad en general con el objetivo de compartir conocimientos, herramientas, formación y recursos sobre ciencia ciudadana.

Cecilia Cabello | Fundación Española para la Ciencia y la Tecnología (FECYT), Ministerio de Ciencia e Innovación

URL de la contribución <http://www.iaph.es/revistaph/index.php/revistaph/article/view/4828>

Si algo caracteriza al siglo XXI es la nueva capacidad de interconexión multidireccional que hemos desarrollado. Desde el inicio de la especie humana hemos creado estrategias de comunicación que nos permitían vivir en comunidad, pero es en la época actual cuando hemos conseguido hacerlo sin importar el tiempo ni los kilómetros que nos separan, difuminándose cada vez más las líneas que separan los diferentes sectores de opinión y decisión.

A lo largo de las últimas décadas se ha reivindicado la importancia de incluir a la ciudadanía en los procesos científicos, no como objetos de estudio, sino como sujetos activos de la investigación. Siguiendo la estela de comunidades creadas alrededor de un tema concreto, -conocido es el caso de la revista de botánica del siglo XIX The Gardeners' Chronicle, que ayudó a científicos como Darwin en sus investigaciones- internet nos ha servido para que cualquier investigador o investigadora pueda plantear sus preguntas abiertamente y recibir la ayuda que necesita para resolverlas.

Las cosas, aunque ya existan, toman relevancia cuando se les da nombre, y a estos procesos se les llama ciencia ciudadana. Francisco Sanz, director de la Fundación Ibercivis, apuntaba que "cuando tú estés estudiando cómo reducir el impacto ambiental de los residuos que produces estarás haciendo, aunque no lo sepas, ciencia ciudadana. Cuando, junto a tu asociación de vecinos, analices la calidad del agua de tu barrio, o te acerques a tu ayuntamiento a pedir los informes al respecto, estarás haciendo ciencia ciudadana" (Sanz 2020). La visión de la ciencia ciudadana consiste en implicar a la sociedad en la investigación científica para generar nuevo conocimiento o comprensión de un tema concreto que, además, tenga potencial de impactar no sólo en la investigación, sino también en el desarrollo de políticas públicas.

El éxito de este tipo de actividades en comunidad requiere a su vez una organización interconectada, una nueva comunidad que integre a las otras más pequeñas. La ciencia ciudadana no se entiende como un punto, sino como un conjunto y, por este motivo, la Fundación Española para la Ciencia y la Tecnología (FECYT), junto al Ministerio de Ciencia e Innovación, ha impulsado en los últimos años un plan de fortalecimiento para el desarrollo y consolidación de la ciencia ciudadana en España como metodología complementaria a la investigación científica. Gracias a sinergias establecidas con entidades como la Fundación Ibercivis, que persigue estos objetivos sin ánimo de lucro, se han desarrollado iniciativas como el Foro Internacional de Ciencia Ciudadana o participado en proyectos de impacto.

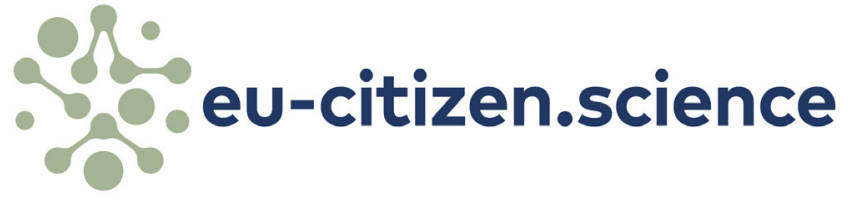




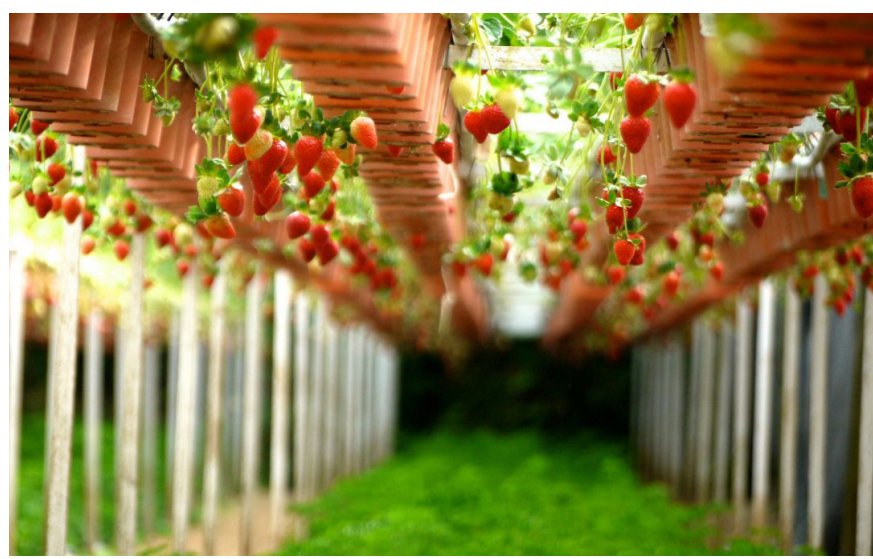

Proyecto Vigilantes del aire | foto FECYT

La intención de convertir España en un punto de referencia de la ciencia ciudadana, y servir como ejemplo para el desarrollo de medidas y políticas que promuevan esta manera de hacer ciencia, se ve reflejada en casos de éxito como la línea de financiación de FECYT específica para proyectos de ciencia ciudadana a través de su convocatoria de ayudas para el fomento de la cultura científica, tecnológica y de la innovación. Un ejemplo de ello es el desarrollo del Observatorio de la Ciencia Ciudadana en España o proyectos como Vigilantes del aire, donde, a partir de algo simple y atractivo como plantar una fresa en nuestros balcones, se ha conseguido describir la calidad del aire en nuestras ciudades y guiar la toma de decisiones y el establecimiento de medidas públicas para mejorarla.

Casos como este, y gracias a la publicación del White Paper on Citizen Science (Comisión Europea 2015), surgió una demanda a nivel europeo para que las iniciativas de ciencia ciudadana existentes se interconectasen más allá de las fronteras de cada país. Fue así como nació el proyecto EU.CITIZEN.SCIENCE, financiado por el Programa Horizonte 2020 de la Comisión Europea a través de su línea de trabajo "Ciencia con y para la Sociedad" (SwafS, por sus siglas en inglés). Dirigido a profesionales de dicho ámbito, personal investigador, responsables políticos y sociedad en general, el objetivo de este proyecto es crear una plataforma online que permita compartir conocimientos, herramientas, formación y recursos sobre ciencia ciudadana para transformar la forma en la que se concibe y desarrolla la investigación científica en la que la ciudadanía participa activamente.

FECYT y otras 23 entidades de hasta trece países europeos tenemos ahora la misión de convertir a la plataforma en un espacio de referencia para el apoyo y desarrollo de esta nueva metodología científica.

La ciencia ciudadana nos sirve para observar y analizar los problemas e inquietudes de la sociedad actual. Como decía Rosalind Franklin, "la ciencia y la vida del día a día no pueden ni deben estar separadas", y por eso es importante implicar a la sociedad en la actividad científica. Como sociedad necesitamos tener la capacidad de discernir lo que es real y lo falso, de entender objetivamente nuestra realidad. Una ciencia hecha en comunidad nos alineará con los Objetivos de Desarrollo Sostenible y es un primer paso para decidir cómo queremos construir nuestro futuro.

\section{BIBLIOGRAFÍA}

- Comisión Europea (2015) White Paper on Citizen Science. Disponible en: https://ec.europa.eu/futurium/en/content/whitepaper-citizen-science [Consulta: 13/01/2021]

- FECYT (2020) Nace la plataforma europea de ciencia ciudadana EU-Citizen.Science. Youtube, 26 de octubre de 2020. Disponible en: https://www.youtube.com/watch?v=TD53_ YEyuOc\&feature=emb_logo [Consulta: 13/01/2021]

- Sanz, F. (2020) Ciencia Ciudadana y Objetivos de Desarrollo Sostenible. En: Observatorio de la Ciencia Ciudadana en España. Fundación Ibercivis, Fundación Española para la Ciencia y la Tecnología (FECYT)-Ministerio de Ciencia e Innovación. Disponible en: https://ciencia-ciudadana.es/ ciencia-ciudadana-y-objetivos-de-desarrollo-sostenible/ [Consulta: 13/01/2021] 